\title{
Shengmai San Alleviates Diabetic Cardiomyopathy Through Improvement of Mitochondrial Lipid Metabolic Disorder
}

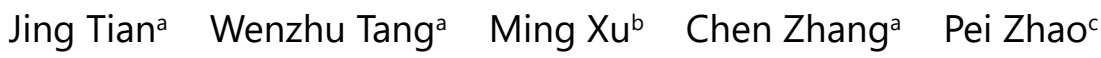 \\ Tongtong $\mathrm{CaO}^{c}$ Xiaoli Shanc Rong Lu ${ }^{\mathrm{a}}$ Wei Guo \\ a'Department of Pathology, Shanghai University of Traditional Chinese Medicine, Shanghai, bDepartment \\ of Physiology, Shanghai University of Traditional Chinese Medicine, Shanghai, 'The Public Experiment \\ Platform, Shanghai University of Traditional Chinese Medicine, Shanghai, China
}

\section{Key Words}

Shengmai San - Diabetic cardiomyopathy - Mitochondria - Mice - Peroxisome proliferatoractivated receptor gamma coactivator 1 -alpha

\begin{abstract}
Background/Aims: Shengmai San (SMS), prepared from Panax ginseng, Ophiopogonjaponicus, and Schisandra chinensisin, has been widely used to treat ischemic disease. In this study, we investigated whether SMS may exert a beneficial effect in diabetic cardiomyopathy through improvement of mitochondrial lipid metabolism. Methods: A leptin receptor-deficient $d b / d b$ mouse model was utilized, and lean age-matched C57BLKS mice served as non-diabetic controls. Glucose and lipid profiles, myocardial structure, dimension, and function, and heart weight to tibial length ratio were determined. Myocardial ultrastructural morphology was observed with transmission electron microscopy. Protein expression and activity of oxidative phosphorylation (OXPHOS) complex were assessed using western blotting and microplate assay kits. We also observed cellular viability, mitochondrial membrane potential, OXPHOS complex activity, and cellular ATP level in palmitic acid-stimulated H9C2 cardiomyocytes. Changes in the sirtuin 1 (SIRT1)/AMP-activated protein kinase (AMPK)/peroxisome proliferator-activated receptor gamma coactivator 1 -alpha (PGC-1 $\alpha$ ) pathway and mitochondrial uncoupling signaling were assessed using western blotting and quantitative real-time PCR. Results: Leptin receptordeficient $d b / d b$ mice exhibit obesity, hyperglycemia, and hyperlipidemia, accompanied by distinct myocardial hypertrophy and diastolic dysfunction. SMS at a dose of $3 \mathrm{~g} / \mathrm{kg}$ body weight contributed to a recovery of diabetes-induced myocardial hypertrophy and diastolic dysfunction. SMS administration led to an effective restoration of mitochondrial structure and function both in vivo and in vitro. Furthermore, SMS markedly enhanced SIRT1 and p-AMPK $\alpha$ protein levels and decreased the expression of acetylated-PGC-1 $\alpha$ and uncoupling protein 2 protein. SMS also restored the depletion of NRF1 and TFAM levels in diabetic hearts and

J. Tian and W. Tang contributed equally to this work.

Wei Guo and Rong Lu

Department of Pathology, Shanghai University of Traditional Chinese Medicine,

1200 Cailun Road, Shanghai 201203 (China)

Tel. +86-21-51322158, E-Mail weiguo@shutcm.edu.cn; lurong@shutcm.edu.cn
\end{abstract}


H9C2 cardiomyocytes. Conclusion: The results indicate that SMS may alleviate diabetesinduced myocardial hypertrophy and diastolic dysfunction by improving mitochondrial lipid metabolism.

\section{Introduction}

Type 2 diabetes mellitus (T2DM) is associated with an increased risk of cardiovascular disease [1]. Diabetic cardiomyopathy (DCM), a leading cause of morbidity and mortality, is a diabetes-related complication characterized by left ventricular (LV) hypertrophy, myocardial fibrosis, and compromised myocardial function $[2,3]$. Though DCM has been strongly linked to metabolic disorders such as increased lipid oxidation and reduced glucose utilization, the exact pathophysiologic mechanism has not been fully elucidated. Cumulative evidence demonstrates that mitochondrial dysfunction is tightly associated with DCM. Mitochondria isolated from atrial tissue of diabetic patients exhibit reduced respiratory function compared with mitochondria from non-diabetic subjects [4]. Hearts from rodent models of T2DM show reduced mitochondrial capacity and contractile dysfunction $[5,6]$. Thus improvement of mitochondrial function has emerged as a promising therapeutic strategy for DCM.

Shengmai San (SMS) is a compound medication including Panax ginseng, Ophiopogon japonicus, and Schisandra chinensisin. In Asia, SMS is typically used in ischemic diseases mainly to treat oxidative stress and inflammation [7-9]. Growing evidence suggests that some of the components of SMS, ginseng and ginsenosides, inhibit adipogenesis and maintain glucose homeostasis in obese and diabetic individuals $[10,11]$. A polysaccharide from Ophiopogon japonicus was found to alleviate hyperlipidemia in diet-induced obese mice based on the metabolic profile of bile acids [12]. However, the exact effect and potential mechanisms of SMS on DCM remain unclear.

In our previous study, we demonstrated that SMS alleviated myocardial fibrosis in aged T2DM via reducing pro-fibrotic proteins [13], and the finding was supported by the results of another study using a rat T2DM model induced by a high-fat diet and streptozotocin injection [14]. Recently, a proteomic study revealed that SMS derived injection modulated a series of energy metabolic proteins associated with glucose oxidation, the tricarboxylic acid cycle, and ATP synthesis in rat hearts subjected to ischemia-reperfusion [15]. Based on the strong correlation between DCM and aberrant mitochondria, we speculated that SMS may exert a beneficial role in DCM by improving mitochondrial lipid metabolism. In the present study, we used a leptin receptor-deficient $d b / d b$ mouse model and a palmitic acid-stimulated H9C2 cardiomyocyte model to investigate the effects of SMS on diabetic cardiomyopathy.

\section{Materials and Methods}

\section{Drugs and reagents}

SMS was composed of Panax ginseng, Ophiopogon japonicus, and Schisandra chinensis in a dry weight ratio of 1:3:1.5. The mixture was boiled, concentrated using a rotary evaporator, and lyophilized using an ALPHR 1-2 LD plus freeze dryer (Martin Christ Gefriertrocknungsanlagen GmbH, Osterode, Germany), and the extracted powder was dissolved in distilled water before use. ATP, ADP, AMP, free fatty acid (FFA) quantitation kit, palmitic acid, and carbonyl cyanide 3-chlorophenylhydrazone were purchased from SigmaAldrich (St. Louis, MO). Mitochondrial isolation and oxidative phosphorylation complex activity kits were purchased from Abcam (Cambridge, UK). Dulbecco's modified Eagle's medium, fetal bovine serum, penicillin, and streptomycin were obtained from GIBCO (Carlsbad, CA). A Cell Counting Kit-8 (CCK-8) and Enhanced ATP Assay Kit were purchased from Beyotime Biotechnology (Nanjing, China). JC-1 was purchased from Invitrogen (Carlsbad, CA). 


\section{Cellular Physiology Cell Physiol Biochem 2018;50:1726-1739 and Biochemistry \begin{tabular}{l|l} 
DOI: 10.1159/000494791 & O 2018 The Author(s). Published by S. Karger AG, Basel \\
Published online:2 November 2018 & www.karger.com/cpb
\end{tabular} \\ Tian et al.: Shengmai San Alleviates Diabetic Cardiomyopathy}

\section{Animals and treatment}

Male 8-week-old leptin receptor-deficient $d b / d b$ mice (BKS.Cg+/+Leprdb NJU) and non-diabetic mice (C57BLKS) were purchased from the Model Animal Research Center of Nanjing University (Nanjing, China). All experimental protocols were approved by the Animal Care and Use Committee of Shanghai University of Traditional Chinese Medicine (Shanghai, China). Prior to the experiments, all mice were given a period of about 10 days for acclimatization, then $d b / d b$ mice were randomly divided into three groups: $d b / d b$ (no SMS), $d b / d b$ plus SMS $3 \mathrm{~g} / \mathrm{kg}$ body weight, and $d b / d b$ plus SMS $4.5 \mathrm{~g} / \mathrm{kg}$ body weight. Lean age-matched C57BLKS mice served as non-diabetic controls. Mice in the non-diabetic control and $d b / d b$ alone groups were treated with double-distilled water, once a day by oral gavage for 2 months.

Fasting blood glucose and intraperitoneal glucose tolerance test (IP-GTT) assay

Blood samples were collected from the tail vein of mice fasted overnight and measured using a digital blood glucose meter (Optium Xceed, Abbott Laboratories, Chicago, IL). The IP-GTT assay was performed with intraperitoneal injection of $1 \mathrm{~g} / \mathrm{kg}$ glucose to assess glucose tolerance. Blood glucose concentration was determined during a 2-h post-injection period $(30,60,90$, and $120 \mathrm{~min})$ and presented as the area under the curve.

\section{Plasma lipid metabolic profile}

At the end of the experiment, blood was collected from the abdominal aortic vein of mice fasted overnight. Collected blood was centrifuged at $3000 \times g\left(4^{\circ} \mathrm{C}\right)$ for $10 \mathrm{~min}$, and plasma triglyceride, total cholesterol, and low-density lipoprotein-cholesterol levels were measured using an automatic biochemical analysis system (Hitachi, Tokyo, Japan). Plasma FFA content was measured with a fatty acid quantitation kit (Sigma-Aldrich) using a colorimetric assay at $570 \mathrm{~nm}$.

Evaluation of cardiac dimensions and function in vivo

To evaluate cardiac dimensions and function, mice were anesthetized with isoflurane ( $3 \%$ induction and 1\% maintenance) and underwent echocardiographic examinations using a high-resolution Vevo 2100 micro-imaging system (Visual Sonics, Toronto, Canada). Cardiac dimensions and function were evaluated by M-mode, pulse-wave and tissue Doppler in mice at baseline and after various treatments.

\section{Determination of heart weight (HW) to tibia length (TL) ratio}

Body weight, TL, and HW were measured in mice at the end of the experiments. Hearts were harvested, rinsed briefly in phosphate-buffered saline (PBS), blotted dry, and weighed. HW was normalized to TL to assess changes in hypertrophy.

\section{Histologic analysis using hematoxylin and eosin (H\&E) staining}

Tissue samples were fixed in $4 \%$ paraformaldehyde overnight, embedded in paraffin, and serially sectioned at a thickness of $5 \mu \mathrm{m}$ for histologic analysis. H\&E staining was carried out following standard procedures, then the sections were investigated using an optical microscope (Stemi DV4 or Axio Scope A1, Carl Zeiss, Oberkochen, Germany).

\section{Observation of mitochondrial structure}

The heart tissues were sliced and fixed with $2.5 \%$ glutaraldehyde in PBS at $4{ }^{\circ} \mathrm{C}$ overnight, and then fixed with $1 \%$ osmium tetroxide in PBS for $2 \mathrm{~h}$. The sliced hearts were stained with uranyl acetate and lead citrate and observed with transmission electron microscopy (Philips Electronic Instruments, Amsterdam, the Netherlands).

\section{Evaluation of mitochondrial function in vivo}

Total oxidative phosphorylation (OXPHOS) complex protein expression

Heart tissues were lysed using standard procedures, and the protein content was determined by bicinchoninic acid (BCA) assay. Total OXPHOS protein expression (complexes I, II, III, IV, and V) was determined by western blotting. 


\section{Cellular Physiology Cell Physiol Biochem 2018;50:1726-1739

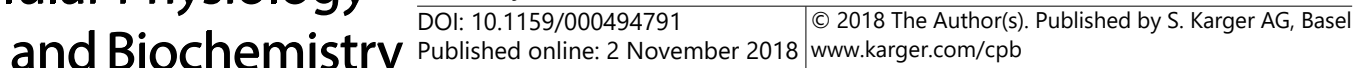

Isolation of cardiac mitochondria: Cardiac mitochondria were isolated using a mitochondrial isolation kit. Protein was quantified by BCA assay.

OXPHOS complex activity assay: Respiratory complex activities (complexes I and IV) from isolated mitochondria were assessed using microplate assay kits (ab109721, ab109911; Abcam).

Cardiac energy charge (EC): To investigate the energy status, fresh myocardium was rapidly homogenized in $200 \mu \mathrm{l} \mathrm{4.2 \%} \mathrm{KClO}_{4}$ and centrifuged at $3000 \mathrm{rpm}$ for $15 \mathrm{~min}$ at $4{ }^{\circ} \mathrm{C}$. The supernatant was added to $80 \mu \mathrm{l} 1 \mathrm{M} \mathrm{NaOH}$ and centrifuged at $8000 \mathrm{rpm}$ for $10 \mathrm{~min}$ at $4{ }^{\circ} \mathrm{C}$. ATP, ADP, and AMP content were determined by high-performance liquid chromatography (HPLC; Agilent Technologies, Santa Clara, CA); a diamonsil plus C-18 $250 \times 4.6 \mathrm{~mm}$ column was used. ATP, ADP, and AMP content were determined using standard curves. EC was calculated according to the following equation: $E C=(A T P+A D P / 2) /(A T P+A D P$ + AMP).

\section{Western blot analysis}

Heart tissues or cells were lysed using standard procedures, and the protein content was determined by BCA assay. Protein bands were detected using a Fluor Chem E imaging system (ProteinSimple, San Francisco, CA). Quantitation was performed using Image J software (National Institutes of Health, Bethesda, MD). $\beta$-actin was used as the loading control for total protein expression. The following primary antibodies were used: anti-acetyl lysine, Total OXPHOS Rodent WB Antibody, anti-peroxisome proliferator-activated receptor gamma coactivator 1-alpha (PGC-1 $\alpha$; all Abcam), anti-sirtuin 1 (Sirt1), anti-phospho-AMP-activated protein kinase (AMPK) $\alpha$ (Thr172), anti-AMPK $\alpha$, anti-NRF1, anti-TFAM (all Cell Signaling Technology, Danvers, MA), and anti- $\beta$-actin (Santa Cruz Biotechnology, Santa Cruz, CA).

\section{RNA extraction and quantitative real-time PCR}

Total RNA was isolated from frozen LV tissue using Trizol reagent (Invitrogen), and reverse transcribed to cDNA using the PrimeScript RT reagent kit with gDNA Eraser (Takara Bio, Kusatsu, Japan), then real-time PCR was performed using SYBR green (Takara Bio). Amplification was monitored using the Light Cycler 96 Real-Time PCR system (Roche, Basel, Switzerland). The following primer sequences in Table 1 were used.

\section{Cell culture and treatment}

To further explore the protective effects of SMS against diabetic cardiomyopathy, we treated H9C2 cardiomyocytes with bovine serum albumin (BSA)-conjugated palmitic acid (C16:0) to mimic lipid overload. H9C2 cardiomyocytes was purchased from Shanghai Institute of Cell Biology (Shanghai, China). Palmitic acid was prepared as a $20 \mathrm{mM}$ solution with $1 \mathrm{ml}$ dissolved in $0.1 \mathrm{M} \mathrm{NaOH}$ at $65-70{ }^{\circ} \mathrm{C}$, and then mixed

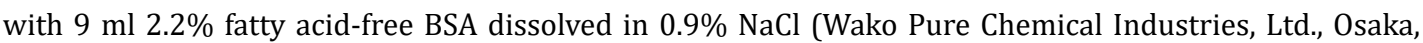
Japan; cat. 013-15143). The conjugated solution was sonicated briefly, incubated for $15 \mathrm{~min}$ at $55^{\circ} \mathrm{C}$, and then cooled to $37^{\circ} \mathrm{C}$. This gave a final molar ratio of FFA/BSA of approximately $6: 1$ in the stock solution as previously described [16-18]. Cells were exposed to $200 \mu \mathrm{M}$ palmitic acid with or without SMS for $24 \mathrm{~h}$, and the same concentration of BSA as control.

\section{Cell viability assay}

H9C2 cell viability was determined using the CCK-8. After treatment, $10 \mu \mathrm{l}$ CCK-8 solution was added and incubated at $37^{\circ} \mathrm{C}$ for $90 \mathrm{~min}$, and the absorbance of each well at $450 \mathrm{~nm}$ was measured using a Synergy2 Multi-Mode Microplate Reader (BioTek Instruments, Inc., Winooski, VT).

\section{Mitochondrial membrane potential}

After treatment, H9C2 cells were washed twice with PBS and incubated with $2.5 \mu \mathrm{M} \mathrm{JC}-1$ for 20 min at $37^{\circ} \mathrm{C}$. The 96 -well plate was then loaded immediately on the Synergy2 Multi-Mode Microplate Reader. The fluorescence intensity was measured with an excitation wavelength of $485 \mathrm{~nm}$, and dual emission filters of $528 \mathrm{~nm}$ (green) and $590 \mathrm{~nm}$ (red). A decrease in $\mathrm{red} /$ green ratio is an indication of reduced mitochondrial membrane

Table 1. Primers used for qRT PCR

\begin{tabular}{lcc}
\hline & Forward primer sequence & Reverse primer sequence \\
\hline 18s & GTAACCCGTTGAACCCCATT & CCATCCAATCGGTAGTAGCG \\
nrf1 & GCACCTTTGGAGAATGTGGT & GGGTCATTTTGTCCACAGAGA \\
tfam & CCTTCGATTTTCCACAGAACA & GCTCACAGCTTCTTTGTATGCTT \\
\hline
\end{tabular}




\section{Cellular Physiology Cell Physiol Biochem 2018;50:1726-1739 \begin{tabular}{l|l|l} 
and Biochemistry Published online: 2 November 2018 & $\begin{array}{l}\text { (c) } 2018 \text { The Author(s). Published by S. Karger AG, Basel } \\
\text { www.karger.com/cpb }\end{array}$ \\
\hline
\end{tabular} \\ Tian et al.: Shengmai San Alleviates Diabetic Cardiomyopathy}

potential. We also observed changes in mitochondrial membrane potential in cells using a Zeiss LSM880 laser scanning confocal microscope (Carl Zeiss, Thornwood, NY).

Mitochondrial complex activities in vitro and intracellular ATP content

ATP content was measured with a luciferase assay kit (Beyotime Biotechnology). In addition, mitochondrial OXPHOS complex activities in vitro were photometrically determined using mitochondrial isolation and enzyme activity assay kits.

\section{Statistical analysis}

Data were presented as the mean \pm standard error of the mean (SEM). Unpaired $t$-test was used for comparison between two groups. For groups of three or more, the data were subjected to one-way analysis of variance followed by Tukey's post hoc analysis. $P<0.05$ was considered statistically significant.

\section{Results}

T2DM mice exhibited myocardial hypertrophy and diastolic dysfunction

Leptin receptor-deficient $d b / d b$ mice at 10 weeks of age exhibited typical characteristics of T2DM, with an overtly obese phenotype and hyperglycemia (approximately 2- to 3-fold increase in body weight and fasting blood glucose; Fig. 1A and 1B). Of note, there was no significant difference in LV ejection fraction (LVEF) and fractional shortening (LVFS) between non-diabetic and $d b / d b$ mice, suggesting preserved systolic function in the early stage of

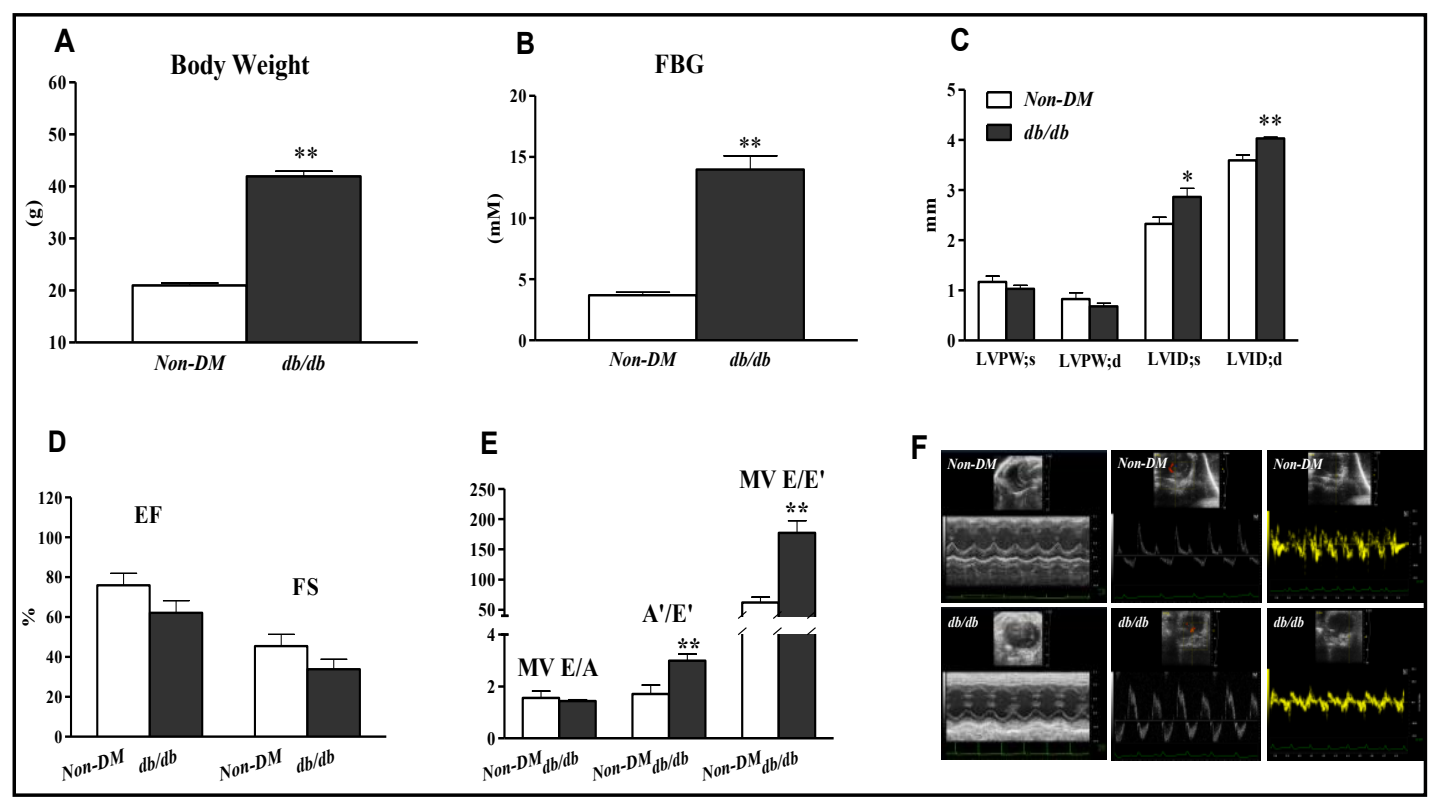

Fig. 1. Type 2 diabetic mice at 10 weeks of age exhibited myocardial diastolic dysfunction with preserved systolic function. (A) Body weight. (B) FBG. (C) Left ventricular dimension measured by echocardiography in anesthetized mice. (D) Left ventricular systolic function assessed by M-mode echocardiography. (E) Left ventricular diastolic function evaluated by pulse-wave and tissue Doppler echocardiography. (F) Representative images of M-mode and Doppler echocardiography. Data are presented as the mean \pm SEM. $\mathrm{n}=5-7$ /group. ${ }^{*} \mathrm{P}<0.05,{ }^{*} \mathrm{P}<0.01$ vs. non-DM group. Abbreviations: FBG, fasting blood glucose. LVPW; s, posterior wall of the left ventricle at systole. LVPW; d, posterior wall of the left ventricle at diastole. LVID; s, left ventricular internal dimension at systole. LVID; d, left ventricular internal dimension at diastole. EF\%, percentage ejection fraction. FS\%, percentage fraction shortening. MV E/A, ratio of early diastolic and atrial trans-mitral inflow velocities. A'/E', ratio of atrial trans-mitral to early diastolic mitral annular velocity. MV E/E', ratio of mitral peak velocity of early filling to early diastolic mitral annular velocity. Non-DM, nondiabetes group. 


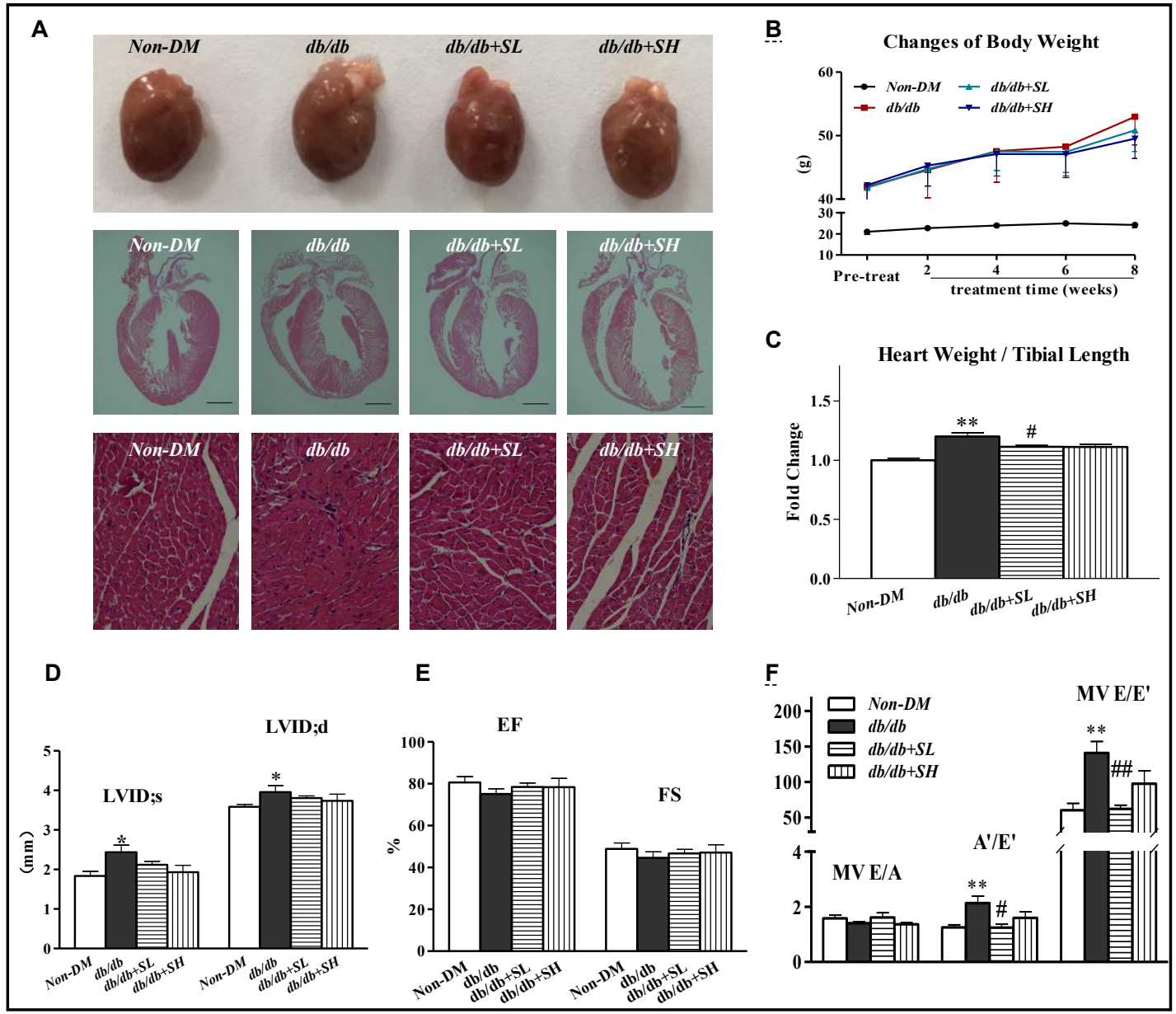

Fig. 2. SMS ameliorated myocardial diastolic dysfunction and hypertrophy in diabetic mice. (A) Representative illustration of whole heart and H\&E staining (magnification: $\times 8$ and $\times 400$, respectively). (B) Changes in body weight during the experimental period. (C) Heart weight/tibial length ratio. (D-F) Left ventricular structural and functional parameters assessed by M-mode and Doppler echocardiography in anesthetized mice. Values are the mean \pm SEM. ${ }^{*} \mathrm{P}<0.05$, ${ }^{* *} \mathrm{P}<0.01$ vs. non-DM group; \#P<0.05, \#\# $\mathrm{P}<0.01$ vs. $\mathrm{db} / \mathrm{db}$ group. Abbreviations: SMS, Shengmai San. SL, SMS (3 g/kg body weight). SH, SMS (4.5 g/kg body weight).

T2DM (Fig. 1C). Echocardiographic results revealed ventricular dilation and cardiac diastolic dysfunction, manifested by enlarged LV inner diameter (LVID) and significantly elevated $\mathrm{A}^{\prime} / \mathrm{E}^{\prime}$ and mitral valve (MV) E/E' ratios. There was a trend toward a decrease in posterior wall thickness and MV E/A (Fig. 1D and 1E). Representative images of M-mode, pulse-wave, and tissue Doppler images are shown in Fig. 1F.

SMS ameliorated diabetes-induced myocardial hypertrophy and diastolic dysfunction

Over the 8-week treatment period, $d b / d b$ mice receiving vehicle alone developed obvious obesity (Fig. 2B) and considerable myocardial hypertrophy, as evidenced by enlarged whole heart, wider LVID during systole and diastole, and elevated HW/TL ratio compared with nondiabetic mice (Fig. 2A-D). H\&E staining showed disordered cardiac fibers, inflammatory cell infiltration, and increased cross-sectional area in cardiomyocytes from $d b / d b$ mice (Fig. 2A). SMS at $3 \mathrm{~g} / \mathrm{kg}$ attenuated the enlargement of the whole heart and increase in HW/TL ratio in $d b / d b$ mice, which was associated with a smaller cross-sectional area in cardiomyocytes than that in $d b / d b$ mice shown in histological images (Fig. 2A and 2C). 


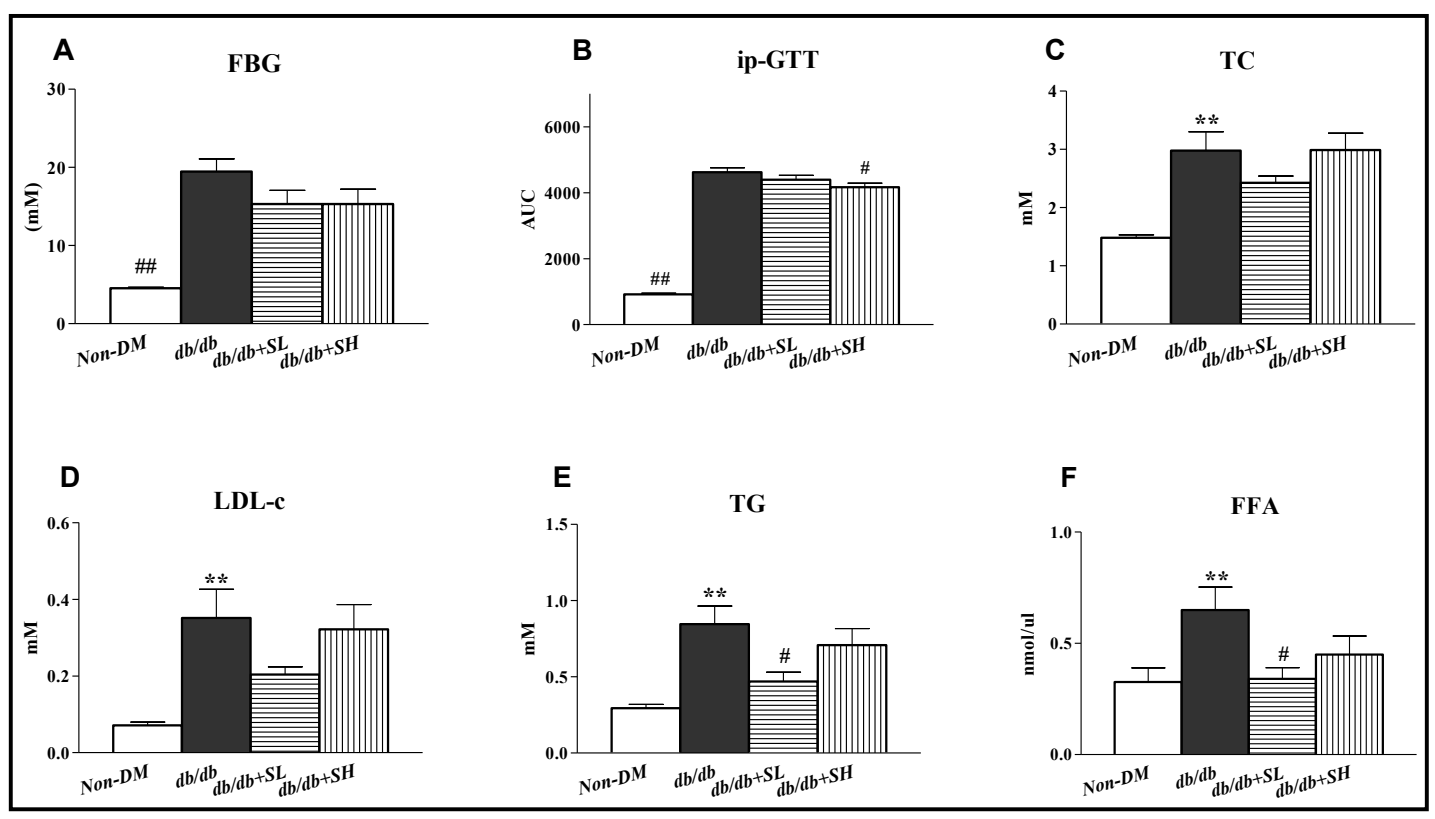

Fig. 3. Effects of Shengmai San (SMS) on metabolic profiles in diabetic db/db mice. (A) Fasting blood glucose (FBG). (B) Intraperitoneal glucose tolerance (IPGTT assay). Briefly, fasted mice were injected with glucose $(1 \mathrm{~g} / \mathrm{kg}$; ip), blood glucose levels were monitored at regular intervals, and data were presented as area under the curve (AUC). (C-F) To assess lipid metabolic profiles, plasma total cholesterol (TC), LDLcholesterol (LDL-c), triglyceride (TG), and free fatty acid (FFA) levels were determined. Values are the mean \pm SEM. $\mathrm{n}=6$-8/group. ${ }^{*} \mathrm{P}<0.05,{ }^{* *} \mathrm{P}<0.01$ vs. non-DM group; $\# \mathrm{P}<0.05$, \#\# $\mathrm{P}<0.01$ vs. $\mathrm{db} / \mathrm{db}$ group.

M-mode, pulse-wave, and tissue Doppler echocardiography were performed in mice after treatment for 8 weeks. No significant difference in LVEF and LVFS were found among the experimental groups, which further supports our finding that systolic function in T2DM could be preserved for a long period. Compared to non-diabetic mice, the $A^{\prime} / E^{\prime}$ and $M V$ $\mathrm{E} / \mathrm{E}^{\prime}$ ratios were clearly elevated in $d b / d b$ mice (Fig. 2E and 2F). SMS at $3 \mathrm{~g} / \mathrm{kg}$ reversed the changes in $A^{\prime} / E^{\prime}$ and MV E/E' ratios in $d b / d b$ mice, indicating that SMS contributes to the recovery of myocardial hypertrophy and diastolic dysfunction.

\section{SMS alleviated metabolic disorder in diabetic mice}

SMS had no obvious hypoglycemic effect in diabetic $d b / d b$ mice, although glucose tolerance was enhanced after a high dose of SMS $(4.5 \mathrm{~g} / \mathrm{kg}$ ) given for 8 weeks (Fig. 3A and 3B). SMS at $3 \mathrm{~g} / \mathrm{kg}$ markedly inhibited the lipid metabolic disorder induced by diabetes (Fig. $3 \mathrm{C}-\mathrm{F}$ ). Triglyceride and FFA levels in $d b / d b+$ SMS (3 g/kg body weight) mice were similar to those in non-diabetic mice. The cardiac lipid accumulation phenotype was further confirmed by transmission electron microscopy, where apparent reduction in lipid droplets was found in non-diabetic and $d b / d b+$ SMS (3 g/kg body weight) hearts (Fig. 4A).

\section{SMS prevented mitochondrial structural damage and dysfunction in diabetic heart}

Myocardial ultrastructural data showed conspicuous mitochondrial injury in diabetic heart, confirmed by larger mitochondria that were more ovoid in shape, with obvious swelling, an extensive loss of cristae, and an excessive distribution of lipid droplets. By contrast, a regular mitochondrial shape and reduced swelling was presented in SMS-treated hearts (Fig. 4A). The protein levels of complexes I, III, and V were significantly decreased in diabetic myocardium, but complex II and IV protein levels were unchanged compared with those in non-diabetic mice (Fig. 4B-D). In addition, inhibition of complex I and IV activity 
A

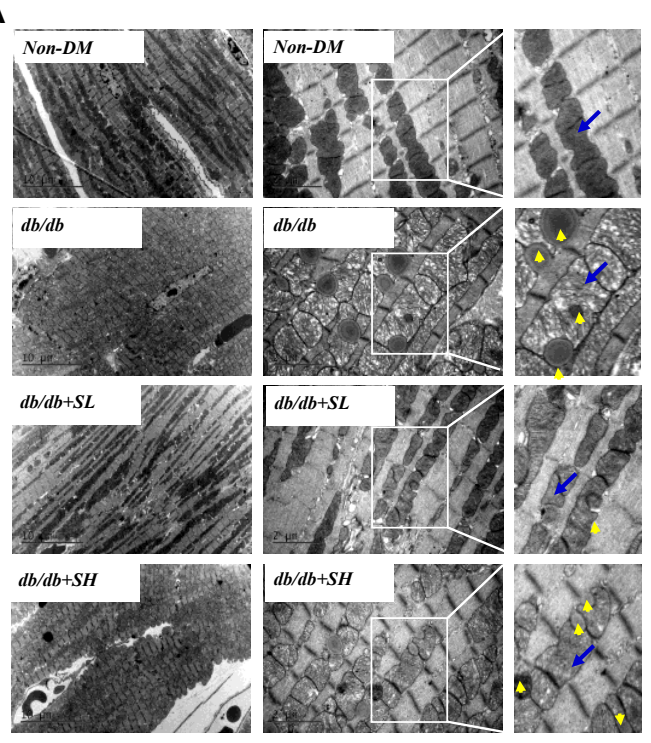

D

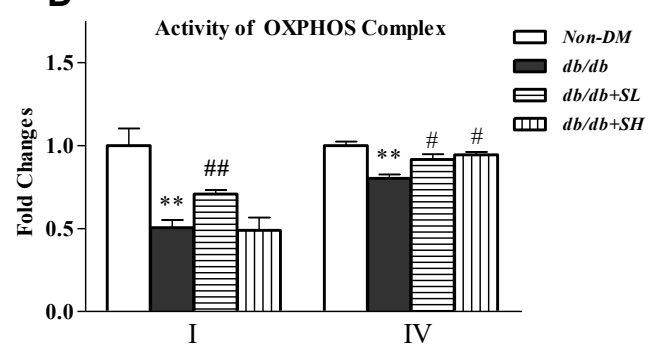

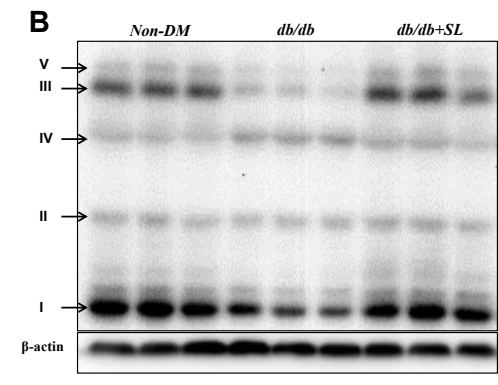

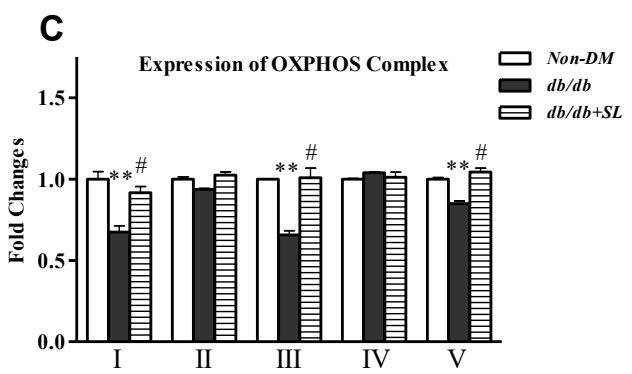

E

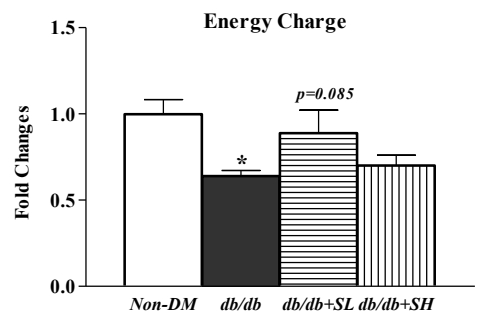

Fig. 4. Myocardial ultrastructural injury and mitochondrial dysfunction in diabetic mice were improved after Shengmai San (SMS) administration. (A) Myocardial ultrastructural changes were observed by transmission electron microscopy. Blue arrows indicate mitochondria; yellow arrows indicate lipid droplets. Scale bar: $10 \mu \mathrm{m}$ (left longitudinal sections) and $2 \mu \mathrm{m}$ (right longitudinal sections). (B, C) Protein expression of mitochondrial OXPHOS complexes I-V determined by western blotting. Myocardial tissues were collected from each group ( $\mathrm{n}=3$ ). (D) Activities of mitochondrial complexes I and IV photometrical measured using microplate assay kits. (E) Values of energy charge (EC) in myocardium calculated as: EC $=(A T P+A D P / 2) /$ (ATP + ADP + AMP). Contents of ATP, ADP, and AMP assayed by high-performance liquid chromatography (HPLC). Values are the mean \pm SEM. ${ }^{*} \mathrm{P}<0.05,{ }^{* *} \mathrm{P}<0.01$ vs. non-DM group; $\# \mathrm{P}<0.05$, \#\# $\mathrm{P}<0.01 \mathrm{vs}$. $\mathrm{db} / \mathrm{db}$ group.

and a decline in EC were also observed in $d b / d b$ hearts (Fig. 4E). SMS had a protective effect against mitochondrial dysfunction in diabetic mice, demonstrated by increased protein levels of complexes I, III, and V (Fig. 4B and 4C) and restoration of the activity of complexes I and IV (Fig. 4D). SMS may protect against DCM through mitochondrial regulation.

\section{Upregulation of SIRT1/AMPK/PGC1 signaling probably contributes to the protective effect} of SMS

Based on the findings described above, we sought to explore the mechanism of mitochondrial restoration conferred by SMS. Western blotting data revealed that SIRT1 and p-AMPK (Thr172) protein levels were reduced in diabetic hearts, accompanied by an increase in acetylated PGC- $1 \alpha$ level (acetylated-lysine site at $110 \mathrm{KDa}$ ), although the expression of PGC- $1 \alpha$ was not altered (Fig. 5A and 5B). Consequently, decreased mRNA and protein expressions of PGC- $1 \alpha$ downstream signals such as NRF1 and TFAM were observed

\section{KARGER}




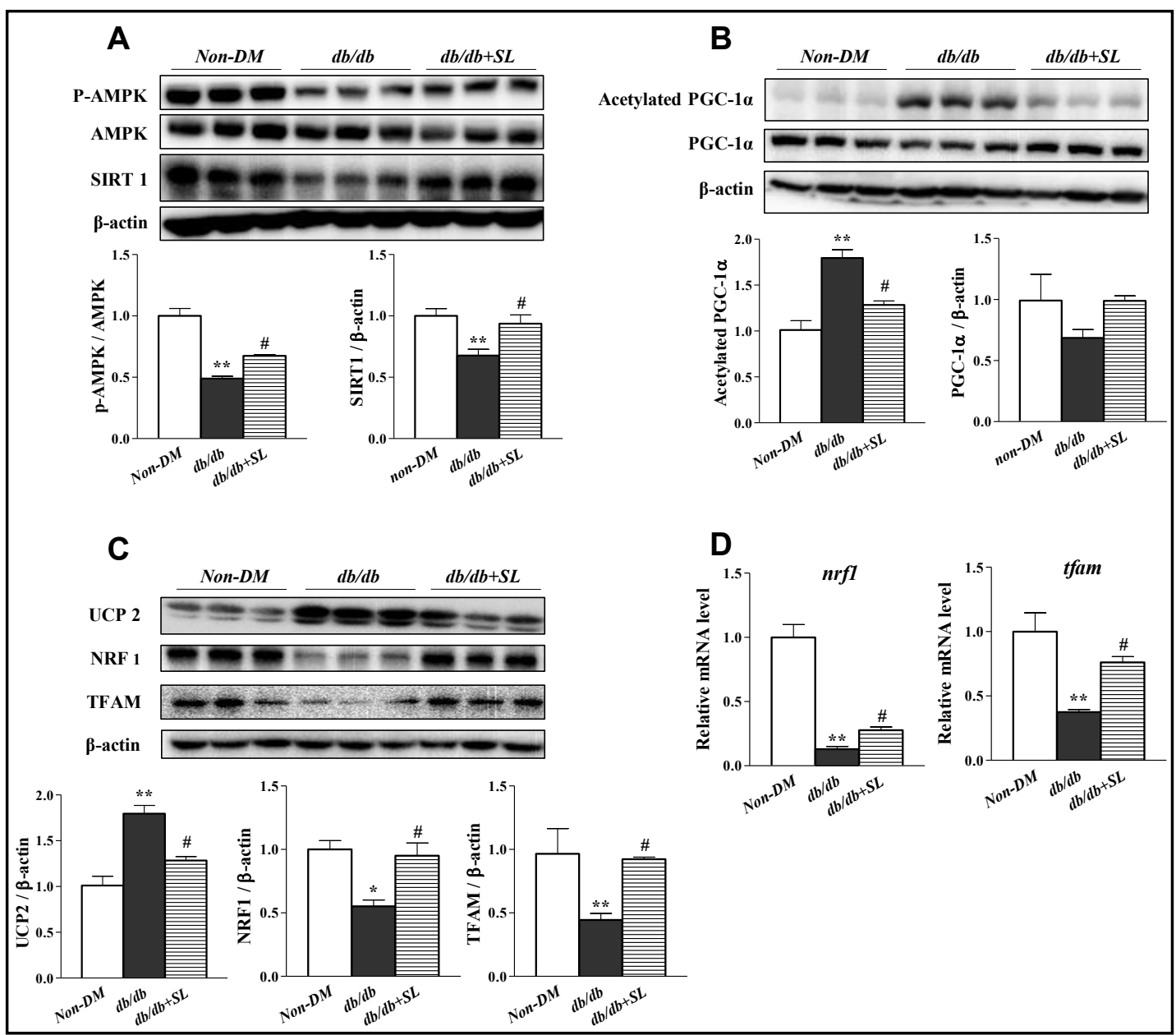

Fig. 5. SIRT1/AMPK/PGC-1 $\alpha$ signal pathway may have a role in the protective effects of SMS. (A-C) Protein expression detected by western blotting. Myocardial tissues were collected from each group $(\mathrm{n}=3)$; bands were normalized relative to $\beta$-actin. (D) Relative mRNA expression of PGC- $1 \alpha$ target gene measured using real-time PCR. Each value was normalized relative to $18 \mathrm{~s}$ mRNA expression. Values are the mean \pm SEM. ${ }^{*} \mathrm{P}<0.05,{ }^{* *} \mathrm{P}<0.01$ vs. non-DM group; $\mathrm{AP}<0.05$, \#\# $\mathrm{P}<0.01$ vs. $\mathrm{db} / \mathrm{db}$ group.

in diabetic hearts (Fig. 5C and 5D). SMS markedly enhanced protein levels of SIRT1 and p-AMPK $\alpha$, and decreased the expression of acetylated PGC- $1 \alpha$ (Fig. 5A and 5B). SMS also restored the depletion of NRF1 and TFAM levels in diabetic heart (Fig. 5C and 5D).

Increased uncoupling protein 2 (UCP2) has been correlated with reduced cardiac efficiency in cardiac hypertrophy in hyperthyroidism and in $d b / d b$ hearts as a result of mitochondrial uncoupling. A similar phenomenon was also present in this study. Furthermore, SMS protected against elevated UCP2 levels in the diabetic heart, suggesting a potential restoration of cardiac efficiency (Fig. 5C).

SMS protected against mitochondrial dysfunction in palmitic acid-induced H9C2 cardiomyocytes

Because SMS improved lipid metabolic disorder rather than decreased blood sugar in diabetic $d b / d b$ mice, we treated H9C2 cardiomyocytes with BSA-conjugated palmitic acid (C16:0) to mimic lipid overload. CCK-8 assay demonstrated a significantly reduced survival in H9C2 cardiomyocytes after palmitic acid stimulation (Fig. 6A). Additionally, palmitic acid contributed to a marked loss of mitochondrial membrane potential, verified by a dramatic decrease in J-aggregate-associated fluorescence (red) and a progressive diffusion 


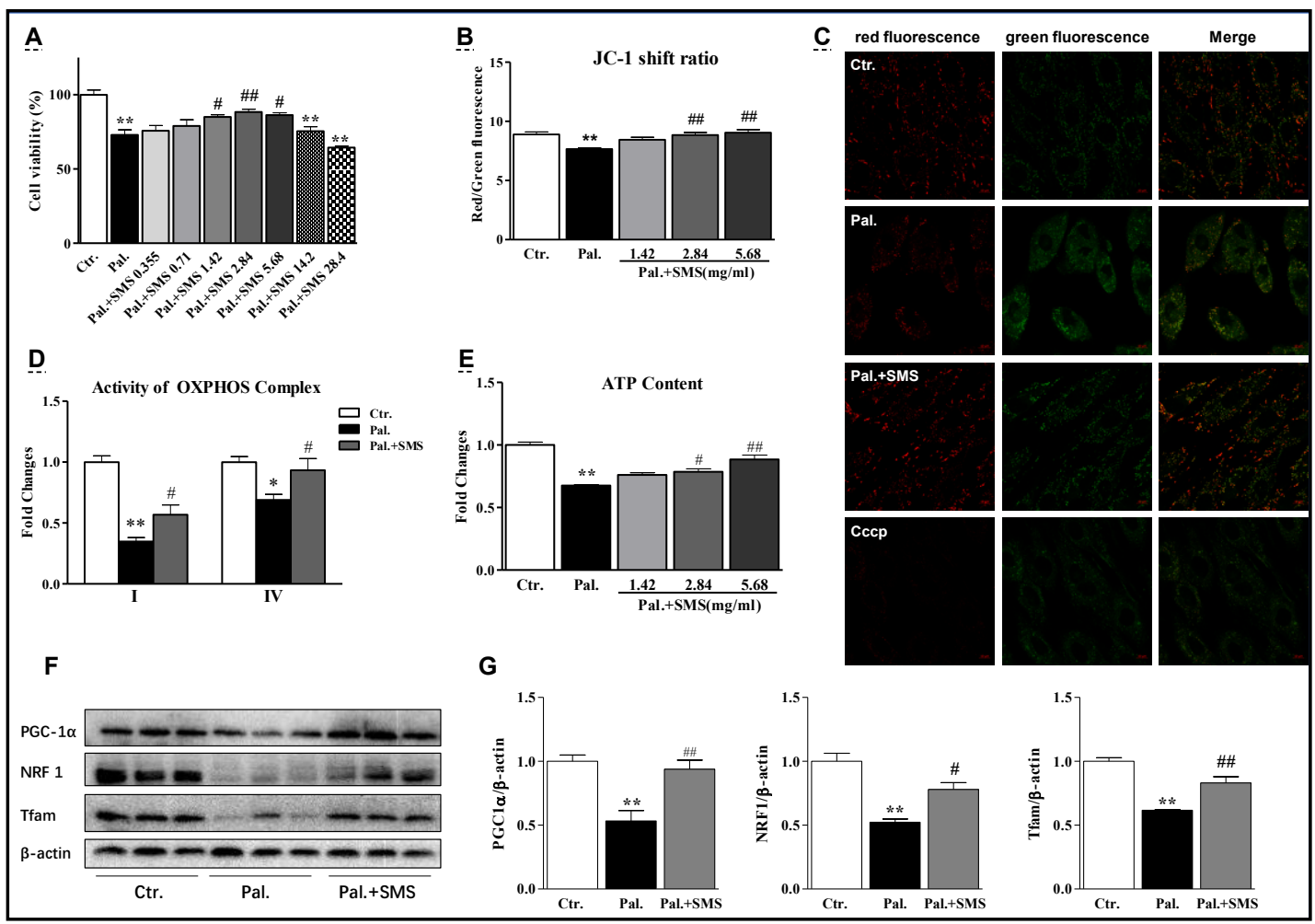

Fig. 6. SMS protected against mitochondrial dysfunction in palmitic acid-induced H9C2 cardiomyocytes. (A) Cell viability. H9C2 cells were exposed to palmitic acid $(200 \mu \mathrm{mol} / \mathrm{l})$ for $24 \mathrm{~h}$ with or without SMS. (B) Changes in mitochondrial membrane potential quantified by the JC-1 shift ratio using a photometric measure. (C) Representative images of mitochondrial membrane potential using confocal laser scanning microscopy. Red fluorescence represents JC-1 aggregation and green fluorescence indicates the presence of JC-1 monomer, which enters into the cytosol following mitochondrial membrane depolarization. (D) The activities of mitochondrial complexes I and IV were photometrical measured using microplate assay kits. (E) Cellular ATP content. (F, G) Protein expression from whole cell lysis determined by western blotting. ${ }^{*} \mathrm{P}<0.05,{ }^{*} \mathrm{P}<0.01$ vs. control group; $\# \mathrm{P}<0.05$, \#\# $\mathrm{P}<0.01$ vs. palmitic acid group.

of monomer-associated fluorescence (green). Similar changes occurred in cytoplasm stimulated with carbonylcyanide-3-chlorophenylhydrazone, which acts as a mitochondrial uncoupler (Fig. 6B and 6C). SMS treatment $(2.84$ or $5.68 \mathrm{mg} / \mathrm{ml}$ ) maintained both cellular viability and mitochondrial membrane potential (Fig. 6A-C).

We found that palmitic acid exposure depleted the ATP content and blunted the activity of mitochondrial complexes I and IV in H9c2 cells, whereas SMS treatment largely abrogated these changes, consistent with our findings in vivo (Fig. 6D and 6E). We also determined the expression of the PGC- $1 \alpha$-associated pathway, and found that PGC- $1 \alpha$ and related downstream signals such as NRF1 and TFAM were markedly downregulated in the palmitic acid group, and this was reversed by SMS administration (Fig. 6F and 6G). Our data suggest that SMS can protect against palmitate acid-induced cellular lipotoxicity probably by alleviating mitochondrial dysfunction and regulating the PGC- $1 \alpha$-associated pathway.

\section{Discussion}

In this study, we used the leptin receptor-deficient $d b / d b$ mouse model, a well-accepted model of T2DM. We found that $d b / d b$ mice exhibited obesity, hyperglycemia, hyperlipidemia, and myocardial hypertrophy. Treatment with SMS contributed to a recovery of diabetesinduced myocardial hypertrophy and diastolic dysfunction. SMS markedly inhibited the lipid

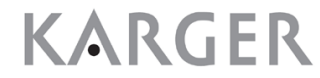




\section{Cellular Physiology Cell Physiol Biochem 2018;50:1726-1739

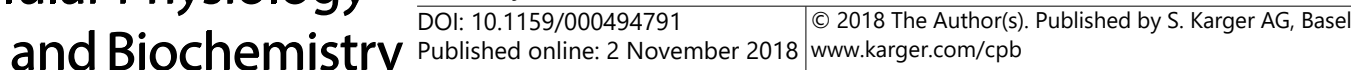 \\ Tian et al.: Shengmai San Alleviates Diabetic Cardiomyopathy}

metabolic disorder induced by diabetes. Plasma triglyceride and FFA levels decreased in SMStreated mice. Additionally, using transmission electron microscopy, we observed that cardiac lipid droplet accumulation and mitochondrial morphology damage were alleviated by SMS. If lipid accumulation, especially toxic lipid intermediates such as ceramides or diacylglycerol, persists over time, it may eventually lead to insulin resistance and compromised myocardial function [19-21]. To further assess the status of mitochondria in vivo, we also measured protein expression and activity of OXPHOS complex, as well as myocardial EC. The protein levels of complexes I, III, and V significantly decreased in diabetic hearts. Additionally, inhibited OXPHOS complex I and IV activities and reduced EC were observed in $d b / d b$ hearts, and these changes were reversed by SMS. These data indicate that SMS exerts a protective effect against DCM through mitochondrial regulation.

The pathophysiologic processes in the diabetic heart are complicated and involve diverse mechanisms, including oxidative stress and inflammation mediated by chronic hyperglycemia, mitochondrial uncoupling, reactive oxygen species/reactive nitrogen species overproduction and endoplasmic reticulum stress triggered by derangement of lipid metabolism, and calcium mishandling and cardiomyocyte apoptosis [22]. To investigate the targets and potential pathways of active components derived from SMS (ginsenoside $\operatorname{Rg} 1$, ginsenoside $\mathrm{Rb} 1$, schisandrin, etc.), Li et al. used a target-pathway interaction network approach and found that the three main signaling pathways were the nuclear factor (NF)$\kappa \mathrm{B}$, oxidative stress, and cytokine network pathways [23]. PGC-1 $\alpha$ is a central regulator of mitochondrial biogenesis and metabolic regulation [24, 25]. PGC-1 $\alpha$ can be activated via various post-translational modifications [26]. AMPK and SIRT1 have been regarded as important upstream mediators of PGC-1 $\alpha$. Both SIRT1-induced deacetylation and direct phosphorylation of PGC- $1 \alpha$ by AMPK lead to marked increases in PGC- $1 \alpha$ activity [27-29]. In the present study, we found that expression of SIRT1 and p-AMPK were reduced in diabetic hearts, accompanied by an increase in acetylated PGC- $1 \alpha$ level. SMS markedly enhanced SIRT1 and p-AMPK $\alpha$ protein levels, and decreased the expression of acetylated PGC-1 $\alpha$ protein. SMS also restored the depletion of NRF1 and TFAM levels in diabetic hearts, which suggests a stabilization and repair of mitochondrial DNA and OXPHOS proteins. These results indicate that SMS mat act through the Sirt1/AMPK/PGC-1 $\alpha$ signaling pathway. Furthermore, SIRT1 can deacetylate pro-inflammatory factor NF- $\mathrm{KB}$ and histone 3 in diabetic rat heart, and attenuate cardiac hypertrophy and oxidative stress [30]. Thus, inhibition of inflammation via SIRT1 and NF- $\kappa \mathrm{B}$, as an early response associated with oxidation, may also have an important role.

Mitra et al. suggested that elevated PGC- $1 \alpha$ in the heart during the early stages of diabetes is probably a response to fatty acid utilization. However, the expression and activity of PGC$1 \alpha$ in diabetes may be reduced as a result of hyperglycemia and lipotoxicity, which gradually induce mitochondrial and myocardial dysfunction [31]. More recently, Diop et al. identified that high-fat diet-induced lipid accumulation and cardiac dysfunction were mimicked by reduced PGC-1/srl function and reversed by PGC-1/srl overexpression [32]. In our study, marked mitochondrial complex defects were observed in diabetic hearts, especially complex I which is a "gatekeeper" of the respiratory chain and catalyzes NADH oxidation to NAD+. We speculate that complex defects may lead to an unbalanced NAD+/NADH ratio and a subsequent decline in NAD+-dependent SIRT1 deacetylation capability. Consistent with this hypothesis, Vazquez et al. reported that mitochondrial complex I defect enhanced protein lysine acetylation in the diabetic heart [33]. Karamanlidis et al. found that protein acetylation resulting from mitochondrial complex I deficiency accelerated heart failure [34].

In vivo, we also detected the expression of mitochondrial UCP2, which has been suggested to be positively associated with reduced cardiac efficiency in $d b / d b$ hearts [35, $36]$. We found that the UCP2 level was higher than in non-diabetic hearts. SMS prevented the elevation of UCP2 in diabetic hearts, which suggests the potential to restore cardiac efficiency. We treated H9C2 cardiomyocytes with palmitic acid to mimic lipid overload in vitro. SMS administration effectively attenuated palmitic acid-induced mitochondrial injury 


\section{Cellular Physiology Cell Physiol Biochem 2018;50:1726-1739 and Biochemistry Published \begin{tabular}{l|l} 
DOI: 10.1159/000494791 2018 The Author(s). Published by S. Karger AG, Basel \\
(c) 2018 \\
www.karger.com/cpb
\end{tabular} \\ Tian et al.: Shengmai San Alleviates Diabetic Cardiomyopathy}

in H9C2 cells, resulting in better preservation of cellular viability, mitochondrial membrane potential, OXPHOS complex activity, and cellular ATP levels.

Impaired glucose metabolism elicited by insulin resistance is widely accepted as a main pathologic trigger in T2DM. Ginsenosides and ophiopogonin from SMS could improve obesity-related insulin resistance and glucose intolerance [37-39]. In the present study, supplementation with a high dose of SMS $(4.5 \mathrm{~g} / \mathrm{kg})$ for 8 weeks enhanced glucose tolerance in diabetic mice, indicating improved insulin sensitivity. However, no obvious lipid-lowering effects were observed in the $4.5 \mathrm{~g} / \mathrm{kg}$ SMS group, and the recovery of cardiac function was not statistically significant. We speculate that SMS at the high dose might promote glucose uptake and utilization, resulting in relatively decreased FFA utilization and unaltered plasma lipid levels.

\section{Conclusion}

In this study, we observed that SMS could alleviate diabetes-induced myocardial hypertrophy and diastolic dysfunction through effective restoration of mitochondrial lipid metabolic disturbance rather than decreasing blood glucose in $\mathrm{db} / \mathrm{db}$ mice. This finding may lead to a better understanding of the role of SMS in DCM and identification of the active components.

\section{Abbreviations}

SMS (Shengmai San); Ctr (bovine serum albumin group); Pal (palmitic acid group).

\section{Acknowledgements}

This work was supported by a grant from the National Natural Science Foundation of China (No. 81473476).

\section{Disclosure Statement}

The authors declare no conflicts of interest.

\section{References}

$>1$ Lorenzo-Almorós A, Tuñón J, Orejas M, Cortés M, Egido J, Lorenzo Ó: Diagnostic approaches for diabetic cardiomyopathy. Cardiovasc Diabetol DOI:10.1186/s12933-017-0506-x.

$>2$ Joshi M, Kotha SR, Malireddy S, Selvaraju V, Satoskar AR, Palesty A, McFadden DW, Parinandi NL, Maulik N: Conundrum of pathogenesis of diabetic cardiomyopathy: Role of vascular endothelial dysfunction, reactive oxygen species, and mitochondria. Mol Cell Biochem 2014;386:233-249.

-3 Falcão-Pires I, Leite-Moreira AF: Diabetic cardiomyopathy: Understanding the molecular and cellular basis to progress in diagnosis and treatment. Heart Fail Rev 2012;17:325-344.

4 Anderson EJ, Kypson AP, Rodriguez E, Anderson CA, Lehr EJ, Neufer PD: Substrate-specific derangements in mitochondrial metabolism and redox balance in atrium of type 2 diabetic human heart. J Am Coll Cardiol 2009;54:1891-1898.

5 Sloan C, Tuinei J, Nemetz K, Frandsen J, Soto J, Wride N, Sempokuya T, Alegria L, Bugger H, Abel ED: Central leptin signaling is required to normalize myocardial fatty acid oxidation rates in caloric-restricted ob/ob mice. Diabetes 2011;60:1424-1434. 


\section{Cellular Physiology Cell Physiol Biochem 2018;50:1726-1739

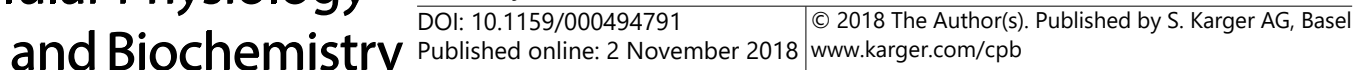

6 Raza H, John A, Howarth FC: Alterations in glutathione redox metabolism, oxidative stress, and mitochondrial function in the left ventricle of elderly zucker diabetic fatty rat heart. Int J Mol Sci 2012;13:16241-16254.

7 Mo WL, Chai CZ, Kou JP, Yan YQ, Yu BY: Sheng-mai-san attenuates contractile dysfunction and structural damage induced by chronic intermittent hypoxia in mice. Chin J Nat Med 2015;13:743-750.

8 Giridharan VV, Thandavarayan RA, Konishi T: Antioxidant formulae, shengmai san, and lingguizhugantang, prevent mptp induced brain dysfunction and oxidative damage in mice. Evid Based Complement Alternat Med DOI: 10.1155/2015/584018.

-9 Zhao Y, Xu L, Qiao Z, Gao L, Ding S, Ying X, Su Y, Lin N, He B, Pu J: Yixin-shu, a shengmai-san-based traditional Chinese medicine formula, attenuates myocardial ischemia/reperfusion injury by suppressing mitochondrial mediated apoptosis and upregulating liver-x-receptor $\alpha$. Sci Rep DOI: 10.1038/srep23025.

10 Li Z, Ji GE: Ginseng and obesity. J Ginseng Res 2018;42:1-8.

$>11$ Li JB, Zhang R, Han X, Piao CL: Ginsenoside rg1 inhibits dietary-induced obesity and improves obesityrelated glucose metabolic disorders. Braz J Med Biol Res DOI: 10.1590/1414-431X20177139.

12 Shi L, Wang J, Wang Y, Feng Y: Mdg-1, an ophiopogon polysaccharide, alleviates hyperlipidemia in mice based on metabolic profile of bile acids. Carbohydr Polym 2016;150:74-81.

13 Zhao J, Cao TT, Tian J, Chen HH, Zhang C, Wei HC, Guo W, Lu R: Shengmai san ameliorates myocardial dysfunction and fibrosis in diabetic $\mathrm{db} / \mathrm{db}$ mice. Evid Based Complement Alternat Med DOI: $10.1155 / 2016 / 4621235$.

14 Ni Q, Wang J, Li EQ, Zhao AB, Yu B, Wang M, Huang CR: Study on the protective effect of shengmai san (see text) on the myocardium in the type 2 diabetic cardiomyopathy model rat. J Tradit Chin Med 2011;31:209219.

15 Zhan S, Fan X, Zhang F, Wang Y, Kang L, Li Z: A proteomic study of shengmai injection's mechanism on preventing cardiac ischemia-reperfusion injury via energy metabolism modulation. Mol Biosyst 2015;11:540-548.

16 Russo SB, Baicu CF, Van Laer A, Geng T, Kasiganesan H, Zile MR, Cowart LA: Ceramide synthase 5 mediates lipid-induced autophagy and hypertrophy in cardiomyocytes. J Clin Invest 2012;122:3919-3930.

17 Mir SUR, George NM, Zahoor L, Harms R, Guinn Z, Sarvetnick NE: Inhibition of Autophagic Turnover in -Cells by Fatty Acids and Glucose Leads to Apoptotic Cell Death. J Biol Chem 2015; 290:6071-6085.

18 Haffara T, Akoumia A, Bousettea N: Lipotoxic palmitate impairs the rate of $\beta$-oxidation and citric acid cycle flux in rat neonatal cardiomyocytes. Cell Physiol Biochem 2016;40:969-981.

19 van de Weijer T, Schrauwen-Hinderling VB, Schrauwen P: Lipotoxicity in type 2 diabetic cardiomyopathy. Cardiovasc Res 2011;92:10-18.

20 Ussher JR: The role of cardiac lipotoxicity in the pathogenesis of diabetic cardiomyopathy. Expert Rev Cardiovasc Ther 2014;12:345-358.

21 Zlobine I, Gopal K, Ussher JR: Lipotoxicity in obesity and diabetes-related cardiac dysfunction. Biochim Biophys Acta 2016;1861:1555-1568.

-22 Jesús FA, Belén P, Elisa R, Jesús E, José T, Óscar L: Targeting metabolic disturbance in the diabetic heart. Cardiovasc Diabetol DOI: 10.1186/s12933-015-0173-8.

-23 Li F, Lv YN, Tan YS, Shen K, Zhai KF, Chen HL, Kou JP, Yu BY: An integrated pathway interaction network for the combination of four effective compounds from ShengMai preparations in the treatment of cardiocerebral ischemic diseases. Acta Pharmacol Sin 2015;36:1337-1348.

24 Patten IS, Arany Z: Pgc-1 coactivators in the cardiovascular system. Trends Endocrinol Metab 2012;23:9097.

25 Palomer X, Salvadó L, Barroso E, Vázquez-Carrera M: An overview of the crosstalk between inflammatory processes and metabolic dysregulation during diabetic cardiomyopathy. Int J Cardiol 2013;168:3160-3172.

-26 Nirwane A, Majumdar A: Understanding mitochondrial biogenesis through energy sensing pathways and its translation in cardio-metabolic health. Arch Physiol Biochem 2018;124:194-206.

27 Dominy JE, Lee Y, Gerhart-Hines Z, Puigserver P: Nutrient-dependent regulation of pgc-1 alpha'sacetylation state and metabolic function through the enzymatic activities of sirt1/gcn5. Biochim Biophys Acta 2010;1804:1676-1683.

-28 Rodgers JT, Lerin C, Haas W, Gygi SP, Spiegelman BM, Puigserver P: Nutrient control of glucose homeostasis through a complex of pgc-1 $\alpha$ and sirt1. Nature 2005;434:113-118. 


\section{Cellular Physiology Cell Physiol Biochem 2018;50:1726-1739

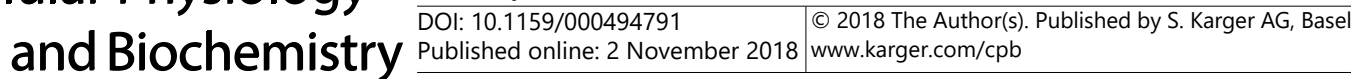 \\ Tian et al.: Shengmai San Alleviates Diabetic Cardiomyopathy}

29 Wu H, Deng X, Shi Y, Su Y, Wei J, Duan H: Pgc-1 $\alpha$, glucose metabolism and type 2 diabetes mellitus. J Endocrinol 2016;229:R99-R115.

-30 Bagul PK, Deepthi N, Sultana R, Banerjee SK: Resveratrol ameliorates cardiac oxidative stress in diabetes through deacetylation of NFkB-p65 and histone 3. J Nutr Biochem 2015;26:1298-1307.

-31 Mitra R, Nogee DP, Zechner JF, Yea K, Gierasch CM, Kovacs A, Medeiros DM, Kelly DP, Duncan JG: The transcriptional coactivators, pgc- $1 \alpha$ and $\beta$, cooperate to maintain cardiac mitochondrial function during the early stages of insulin resistance. J Mol Cell Cardiol 2012;52:701-710.

-32 Diop SB, Bisharat-Kernizan J, Birse RT, Oldham S, Ocorr K, Bodmer R: Pgc-1/spargel counteracts high fat diet-induced obesity and cardiac lipotoxicity downstream of tor and brummer atgl lipase. Cell Rep 2015;10:1572-1584.

-33 Vazquez EJ, Berthiaume JM, Kamath V, Achike O, Buchanan E, Montano MM, Chandler MP, Miyagi M, Rosca MG: Mitochondrial complex I defect and increased fatty acid oxidation enhance protein lysine acetylation in the diabetic heart. Cardiovasc Res 2015;107:453-465.

-34 Karamanlidis G, Lee CF, Garcia-Menendez L, Kolwicz SC, Suthammarak W, Gong G, Sedensky MM, Morgan PG, Wang W, Tian R: Mitochondrial complex i deficiency increases protein acetylation and accelerates heart failure. Cell Metab 2013;18:239-250.

35 Boudina S, Sena S, Theobald H, Sheng X, Wright JJ, Hu XX, Aziz S, Johnson JI, Bugger H, Zaha VG, Abel ED: Mitochondrial energetics in the heart in obesity-related diabetes. Diabetes 2007;56:2457-2466.

-36 Ko TH, Marquez JC, Kim HK, Jeong SH, Lee S, Youm JB, Song IS, Seo DY, Kim HJ, Won DN, Cho KI, Choi MG, Rhee BD, Ko KS, Kim N, Won JC, Han J: Resistance exercise improves cardiac function and mitochondrial efficiency in diabetic rat hearts. Pflugers Arch 2018;470:263-275.

37 Li JB, Zhang R, Han X, Piao CL: Ginsenoside Rg1 inhibits dietary-induced obesity and improves obesityrelated glucose metabolic disorders. Braz J Med Biol Res DOI: 10.1590/1414-431X20177139.

-38 Song B, Ding L, Zhang HQ Chu YF, Chang ZH, Yu YL, Guo DD, Zhang SP, Liu XZ: Ginsenoside Rb1 increases insulin sensitivity through suppressing $11 \beta$-hydroxysteroid dehydrogenase type I. Am J Transl Res 2017;9:1049-1057.

39 Chen SY, Li X, Liu L, Liu C, Han X: Ophiopogonin D alleviates high-fat diet-induced metabolic syndrome and changes the structure of gut microbiota in mice. FASEB J 2018;32:1139-1153. 